\title{
Editorial
}

\section{Development and death of neurons: sealed by a common fate?}

\author{
P Nicotera ${ }^{\star 1}$ \\ ${ }^{1}$ MRC Toxicology Unit, Hodgkin Building, University of Leicester, Lancaster \\ Road, LE1 9HN Leicester, UK \\ * Corresponding author: P Nicotera; E-mail: pn10@le.ac.uk
}

Cell Death and Differentiation (2002) 9, 1277-1278. doi:10.1038/ sj.cdd. 4401162

The father of modern political theory, Niccolò di Bernardo dei Machiavelli (1469-1527) introduced the well-followed principle that Princes should retain absolute control of their territories, and to this end they are entitled to use any means of expediency.

The raison d'être of the neuronal populations within the nervous system is to establish contact with their targets during development and refine it throughout their entire life span. To achieve the scope of forming and retaining a functional nervous system architecture, neuronal differentiation and death are ruthlessly regulated in development and strictly kept under control in post-mitotic systems. A true utilitarian principle governs therefore the harmonic development and survival of the nervous system. Derangements in neural networking would affect many neuronal subpopulations. Therefore individual neurons that fail to retain most of their synaptic connections have no reason to further exist and die. This is accomplished by the activation of several signalling pathways, some of which involve odd players: the molecular components of the developmental cell cycle control. In this issue of Cell Death and Differentiation three reviews and one article are concerned with the mechanisms involved in establishing functional neuronal connections and the possible role of cell cycle regulatory molecules in determining the life and death of neurons.

The olfactory sensory pathway is a unique model to investigate guidance and connectivity of growing axons as well as the cues involved in programmed cell death. The formation of the olfactory bulb involves differentiation of several cell types and extension of central projections. Because there is a close relationship between the olfactory epithelium, the bulb and the olfactory cortex, Drs LopezMascaraque and de Castro ask the question as to whether establishment of the olfactory bulb central projections can proceed independently of the afferent olfactory sensory axons. ${ }^{1}$ This poses another more general question: is establishment of connectivity required to awake a developmental programme in target cells?

According to the authors' own data, the initial establishment of the olfactory bulb central projections can occur independently of the olfactory axons from the olfactory epithelium, which supports the idea that cortical regions are patterned before migration of newborn neurons, at least for this developmental domain. This implies a strict, but intrinsic molecular control of the distinct olfactory structures. How then, do axonal projections find their correct way? Secreted chemo-repellent factors and adhesion molecules may cooperate to prevent axons from invading structures other than the olfactory bulb and, at the same time, stimulate axonal branching in an interplay of attractive and repulsive signals. At later stages, tissue-derived cues will refine the mature appearance of the olfactory bulb. Should this model be correct, it would actually imply the existence of a simultaneous and independent organization of olfactory structures rather than a stepwise process, which depends on established connectivity. Notably, this poses another fundamental question: what keeps the olfactory neurons alive during development of the olfactory structures?

The adult olfactory epithelium is mostly made up of chemosensory neurons, which are very liable to apoptosis and, at difference with other districts, are replaced by permanent neurogenesis from local progenitor cells. Thus, neurogenesis may be a factor which contributes to maintain the number of sensory olfactory neurons prior to development of the olfactory structures. Tissue derived cues may also be very important in deciding death or survival of olfactory neurons even in the absence of established neuronal connections. On the other hand, the sole ablation of the olfactory bulb in the adult (i.e., elimination of the only target for olfactory neurons) is sufficient to cause their massive apoptosis. Dr Meheln and colleagues ${ }^{2}$ have analyzed the genetic response following ablation of the olfactory neurons. They show that apoptosis-related genes are not modified significantly following olfactory bulb ablation, with the possible exception of the tyrosine kinase RIP. This suggests that ablation of the target in this system does not result in the transcriptional regulation of the standard apoptotic signalling pathways. In contrast, the authors find a significant increase in the expression of the serine protease inhibitor, Spi2. SPi2 belongs to the serpin family of proteinase inhibitors. Intracellular serpins normally prevent cell death by inhibiting the caspase execution of apoptosis. ${ }^{3}$ In the study published in this issue, Spi2 seems to be expressed outside the olfactory epithelium, in the underlying lamina propria, which suggests that protease inhibition results in a paracrine signalling leading to neuronal demise. Proteases in the extracellular matrix can indeed be involved in neuronal death in several paradigms of neurodegeneration. Inhibition of certain matrix metalloproteinases $^{4}$ or activation of others by nitrosative stress ${ }^{5}$ has indeed been shown to stimulate neurons to apoptose. 
If the extrinsic cues are all so important for neuronal development and death, which signalling pathways at the cross-road between cell division, differentiation and death decide on these diverging processes within the neuron?

Molecules that may have these all-encompassing features are the cyclin-dependent kinases (Cdks). These cell cycle regulatory molecules can be involved in proliferation senescence and apoptosis. Notably, all Cdks, with the exception of Cdk5 are silenced in post-mitotic neurons and yet they appear to be deregulated in several neurodegenerative conditions, where ectopic expression of these proteins is linked to apoptosis. ${ }^{6}$ The review by $\mathrm{Dr}$ Julien and his colleagues in this issue of $\mathrm{CDD}^{7}$ highlights the multiple facets of Cdk5 and other cycling Cdks. While the levels of cell cycle Cdks is markedly reduced in adult neurons, Cdk5 and its neuronal co-activators p35 and p39 increases. Notably cell cycle Cdks are induced in neuropathological conditions and their expression is linked to de-differentiation and neuronal demise. One of the relevant pathways is the phosphorylation of $\mathrm{Rb}$ by $\mathrm{Cdks},{ }^{8}$ which promotes dissociation of $\mathrm{Rb}$ from E2F-1 and the induction of expression of pro-apoptotic proteins. At difference with cell cycle Cdks, Cdk5 has a direct role in controlling neuronal differentiation and migration as well as a mediator of neuronal death. Cdk5 is involved in neuronal plasticity due to its ability to phosphorylate cytoskeletal components and synaptic or axonal transport proteins. The role of Cdk5 in cell death is more complex and involves potentially different mechanisms. Cdk5 and Cdks may not work concomitantly in the adult CNS, but may cooperate in causing neurodegeneration. Whereas Cdks act primarily by inducing cell cycle and pro-apoptotic genes Cdk5 cause aberrant phosphorylation of cytoskeletal elements, synaptic dysfunction and possibly inhibits anti-apoptotic pathways. Dr. Kroemer and colleagues dedicate special attention to Cdk1 and to its role in apoptosis and mitotic catastrophe. ${ }^{9}$
In non-neuronal cells Cdk1 is activated in several death paradigms including demise caused by aberrant mitosis the 'mitotic catastrophe' and in the cytopathogenicity of HIV-1. Cdk1 may also determine the abnormal resistance to chemotherapy of cancer cells.

While these pathways may not always participate in neuronal demise it is becoming clear that signals leading to neuronal death are often linked to a developmental regression (i.e., loss of projections, cell cycle alterations). Where is then the commitment point in cell death? Death pathways in high organisms have evolved as independent subroutines. Some are restricted to individual tissues as part of their differentiation and developmental programmes, whereas others, which are longer conserved in evolution, are active in every organ and cell type. Each subroutine has a commitment point and often cell death requires the recruitment of many. Neurons may cease to exist when all their relationships are lost and the social marginalisation becomes unbearable, when they regress to an infantile state, when extracellular cues de-repress their death receptors or evict them from territories once hard gained. Their fate is sealed early, as paths are found and established in the struggle for survival.

1. Lopez-Mascaraque L and de Castro F (2002) Cell Death Differ. 9: 1279-1286 (This issue)

2. Thiemmara $V$ et al (2002) Cell Death Differ. 9: 1343-1351 (This issue)

3. Garcia-Calvo M et al (1998) J. Biol. Chem. 273: 32608-32613

4. Ethell D et al (2002) Curr. Biol. 12: 1595

5. Gu Z et al (2002) Science 297: 1186-1190

6. Freeman RS et al (1994) Neuron 12(2): 343-355

7. Nguyen M et al (2002) Cell Death Differ. 9: 1294-1306 (This issue)

8. Johnson DG (1995) Oncogene 11: 1685-1692

9. Castedo M et al (2002) Cell Death Differ. 9: 1287-1293 (This issue) 